Kourosh Ghanadi, Hormoz Mahmoudvand, Ali Bakhtiari, Mojtaba Gorji, Nasroallah Moradi-kor, Mohamad Javad Tarahi, Mahshid Garmsiri, Zia Obeidavi

\title{
Pre-operative laparoscopic staging of gastric cancer in patients who are candidates for neo- adjuvant chemotherapy: A Cross Sectional Study
}

https://doi.org/10.1515/bmc-2019-0008

received December 19, 2018; accepted February 26, 2019.

Abstract: Objective: The aim of this study is to evaluate the laparoscopic method in gastric cancer staging before surgery in patients undergoing Neo-adjuvant chemotherapy.

Methods: This was a cross-sectional study on patients with T3-T4 and node positive non-metastatic gastric adenocarcinoma who was candidates for neo-adjuvant chemotherapy. The patients were excluded from the study when they were not in the condition to continue the study. All eligible patients underwent metastases work up by abdominal, pelvic and chest CT scans and then received neo-adjuvant chemotherapy. Pre-operative laparoscopy was performed before definitive surgery. The collected data was analyzed using SPSS software. The statistical significance, sensitivity, specificity and predictive values were calculated from this data.

Results: In this study, out of 50 patients, 26 (52\%) were male. The mean age of the patients was $62.44 \pm$

\footnotetext{
*Corresponding author: Ali Bakhtiari, Assistant Professor, Department of Internal Medicine, Lorestan University of Medical Sciences, Khorramabad, Iran, E-mail: Dr82658@yahoo.com Kourosh Ghanadi: Associate Professor, Department of Internal Medicine, Lorestan University of Medical Sciences, Khorramabad, Iran

Hormoz Mahmoudvand: Associate Professor, Department of General Surgery, Lorestan University of Medical Sciences, Khorramabad, Iran Mojtaba Gorji: Oncologist, Department of Internal Medicine, Lorestan University of Medical Sciences, Khorramabad, Iran Nasroallah Moradikor: Research Centre of Physiology, Semnan University of Medical Sciences, Semnan, Iran Mohamad Javad Tarahi: Assistant Professor, Department of Epidemiology \& Biostatistics, Isfahan University of Medical Sciences, Isfahan, Iran

Mahshid Garmsiri: Medical Student, Student Research Committee, Lorestan University of Medical Sciences, Khorramabad, Iran Zia Obeidavi: General Physician, Lorestan University of Medical Sciences, Khorramabad, Iran
}

12.136 years. Most tumors were located in one-third distal of stomach (21 patients, 42\%). The accuracy of the laparoscopic method, to determine the degree of involvement of the lymph nodes, revealed that the method had a sensitivity of $84 \%$, a specificity of $47.62 \%$, a positive likelihood ratio (PLR) of 1.6, a negative likelihood ratio (NLR) of $0.33 \%$, a positive predictive value of $65.62 \%$ and a negative predictive value of $71.43 \%$. The accuracy of the laparoscopic method, regarding determining the presence or absence of metastasis, revealed that the method had a sensitivity, specificity, positive predictive value, and a negative predictive value of $100 \%$.

Conclusion: Based on the evidence of this study, and other studies in this field, it seems that the use of a step-bystep combination methods in gastric cancer staging would be logical. Alone, no singular method would be sufficient in providing the physician with sufficient information to successfully stage the tumor and thus determine the appropriate treatment. Therefore, the combination of methods should be used which, in view of the economic constraints of health systems, would be recommended for high-risk patients for metastasis $(\mathrm{N}+/ \mathrm{T} 3-\mathrm{T} 4)$, diagnostic pre-operative laparoscopy.

Keywords: Gastric Cancer; Cross Sectional Study; Laparoscopy; Neoplasm Staging.

\section{Introduction}

Gastric cancer is the second most common cancer around the world with 900,000 new cases diagnosed annually. In Iran, stomach cancer is the second most common cancer (after skin cancer) in men and the fourth most common cancer in women [1]. In recent years, the number of diseased people and the rate of mortality in Iran has increased, following this trend, stomach cancer has the highest mortality rate in Iran [2, 3]. More than $70 \%$ of 
gastric cancers occur in developing countries and are more common in men than in women [4], with a male to female ratio of 1.8-1. The highest prevalence of gastric cancers is in $7^{\text {th }}$ and $8^{\text {th }}$ decades of life [2]. Although the incidence of stomach cancer has declined worldwide, in some regions such as Japan, China, Chile and Ireland, the incidence of this disease remains high [5]. According to the latest National Comprehensive Cancer Network's (NCCN) guideline, the incidence of gastric cancer in China is higher than any other country, and is the most common malignancy in men in Japan. Based on previous studies, smoking, alcohol consumption, Helicobacter pylori infection, and obesity have been identified as environmental risk factors for this disease. However, these factors alone cannot explain the problems associated with high rates of disease; therefore, the risk of hereditary factors should also be considered [2]. In another division, the risk factors of gastric cancer are divided into two groups; the first group has unchangeable factors such as age and sex, and the second group has variable factors, such as smoking and H. Pylori infection [6].

There is currently no unique and gold standard method for gastric cancer staging. In fact, several complementary methods are used in certain clinical conditions [7]. Radiological evaluation (which includes abdominal and pelvic CT scans), laparoscopy, peritoneal cytology and etc., are used in different individuals and conditions for staging. Previously, a 2009 study by Power et al. found that performing EUS prior to laparoscopy improves patient selection [8]. Furthermore, Karanicolas et al. (2011) explored laparoscopic staging in gastric cancer management finding that laparoscopic staging is less common in older patients with stomach adenocarcinoma. However, increasing SL application can lead to reduced mortality and morbidity due to nontherapeutic (non-essential) laparotomy [9]. In another study, Burbidge et al. (2013) evaluated the diagnostic role of CT scan and laparoscopy in the staging of patients with treatable gastric cancer. The researchers emphasize that laparoscopic staging is an essential complement to imaging in all patients who are candidates for gastric cancer surgery [10]. Cameron et al., in their study in 2014, stated that laparoscopic staging can directly lead to a change in treatment in $15 \%$ of patients ( $9 \%$ of gastric tumors and $20 \%$ of esophageal tumors). This is meant to reduce and prevent unnecessary laparotomy.

In fact, diagnostic laparoscopy was first introduced by Kalling in 1901 [11]. This method is more invasive than CT scan and EUS but has the benefit of direct observation of liver surface, peritoneum and local lymph nodes. $20-30 \%$ of patients, with negative CT scan and stage II gastric cancer based on EUS had peritoneal metastasis based on diagnostic laparoscopy. Another advantage of laparoscopy is to create the opportunity for cytology in patients who have no evidence of peritoneal involvement. Currently, CT scans are easily accessible in the Lorestan province and laparoscopy is also possible. In practice, the pathway for diagnosis and treatment is limited to CT scan, endoscopy and pathology and is less commonly used in laparoscopic procedures. Considering the importance of accurate gastric cancer staging in determining the appropriate treatment, avoiding unnecessary measures, and, considering the, ease and inexpensive access to laparoscopy in educational hospitals, the routine use of this method in stomach cancer staging would be logical. Particularly, the exact determination of the stomach cancer stage has led to a reduction in the costs and hospitalization time and mortality of the patients and can provide a reliable therapeutic treatment for the patient. Therefore, in the present study, we evaluated the laparoscopic method in pre-operative gastric cancer staging in patients undergoing Neo-adjuvant chemotherapy, for better management in these patients.

\section{Materials and Methods}

\section{Studied Patients}

This was a cross-sectional study on patients with T3-T4 and node positive non-metastatic gastric adenocarcinoma who was candidates for neo-adjuvant chemotherapy (we select 200 patients and after considering the exclusion criteria, 50 patients entered to the study). The patients were excluded from the study at each stage, in which they did not have the condition to continue the study. These conditions included the detection of metastasis, patient dissatisfaction, and inappropriate clinical conditions.

\section{Procedure}

All eligible patients underwent metastases work up by abdominal, pelvic and chest CT scan and then received neo-adjuvant chemotherapy. Also, pre-operative laparoscopy was performed before definitive surgery. 


\section{Data analysis}

The variables of this study were metastasis (based on laparoscopic findings and pathologic evaluation), lymph node involvement (based on laparoscopic findings and pathologic evaluation), tumor invasion (based on endoscopic ultrasonography and pathologic evaluation), age and gender of the patients. Descriptive statistics and sensitivity, specificity, predictive value and likelihood ratio were used to analyze the information. The statistical software used in this study was SPSS.

\section{Ethics approval and consent to participate}

According to the available sources and the latest available researches, patients with gastric cancer should undergo tumor staging before any treatment. Performing CT scan of the abdomen and pelvis is mandatory and then using other methods based on the results. The necessity or suitability of laparoscopy in patients with T3-T4 stages and multiple visceral involvement is acceptable [12]. The cost of carrying out the above measures was borne by the researchers.

Informed consent: Informed consent has been obtained from all individuals included in this study

Ethical approval: The research related to human use has been complied with all the relevant national regulations, institutional policies and in accordance the tenets of the Helsinki Declaration, and has been approved by the ethics committee of Lorestan University of Medical Sciences.

\section{Results}

In this study, out of 50 patients, 26 (52\%) were male. The mean age of the patients was $62.44 \pm 12.136$ years, the youngest was 36 and the oldest was 87 years old. Most of the patients were from 60 to 69 years old with 16 patients (32\%), 50 to 59 years with 13 patients (26\%) and 70 to 79 years with 11 patients (22\%). Patients under the age of 40 and over 80 (each with 3 patients) had the lowest incidence. Anatomical location of the tumors was also observed, in which 21 patients (42\%) had tumors in onethird distal, 17 patients (34\%) had tumors in one-third proximal and 12 patients (24\%) had tumors in middlethird portion of the stomach. Descriptive results of the status of metastasis (M), lymph node involvement $(\mathrm{N})$ and tumor penetration depth (T) of the patients participating in the study based on two laparoscopic and pathologic methods are presented in Table 1 (Table 1)

Based on another division, the depth of the tumor's penetration are divided into two groups; the first group is low risk (NO/ (T1-T2)) and the second is high risk ( + / (T3-T4)), accordingly in the present study, high-risk cases were reported in 29 patients (58\%) and 9 patients (18\%) based on pathologic and laparoscopic findings and lowrisk cases were reported based on pathologic findings in 17 patients (34\%). The results of laparoscopy have not reported the depth of the tumor in any of the patients at low risk. The accuracy of the results of the laparoscopic method to determine the degree of involvement of the lymph nodes revealed that the method had a sensitivity of $84 \%$, a specificity of $47.62 \%$, a positive likelihood ratio (PLR) of 1.6, a negative likelihood ratio (NLR) of $0.33 \%$, a positive predictive value of $65.62 \%$ and a negative predictive value of $71.43 \%$.

In this study, the results of pathologic studies as a Gold standard were used to determine the accuracy of the results of laparoscopic studies. Accordingly, in the comparative study of the results of lymph node examination with two pathologic and laparoscopic methods, it was determined that the results of the two methods were coincident in 31 $(62 \%)$ and unmatched in $15(30 \%)$ patients. The conformity of these two methods was not detectable in 4 patients (8\%). In the study of the accuracy of the results of the laparoscopic method regarding determining the presence or absence of metastasis, it is revealed that the method had a sensitivity, specificity, positive predictive value, and a negative predictive value of $100 \%$. In a comparative study, the results of the presence or absence of metastasis with two pathologic and laparoscopic methods also revealed that the results of these two methods matched in all 50 patients. In a comparative study, the results of tumor penetration analysis with two pathologic and laparoscopic methods showed that the results of these two methods were matched in $5(10 \%)$ patients and in $2(4 \%)$ patients were non-conforming. The conformity of these two methods was not detectable in 43 patients (86\%).

\section{Discussion}

According to the analytical results of 50 patients, 26 patients (52\%) were male and 24 (48\%) were female. Although the number of sample is low, in general, it indicates that males are more likely to be diseased. This has already been emphasized in previous studies [13-15]. In addition, the mean age of the patients was $62.44 \pm 12.136$ 
Table 1: Descriptive results of the state of metastasis (M), lymph node involvement $(N)$ and tumor penetration depth (T) of the patients participating in the research based on two methods of laparoscopy and pathologic evaluation.

\begin{tabular}{|c|c|c|c|c|c|}
\hline \multirow[t]{2}{*}{ Variables } & & \multicolumn{2}{|l|}{ pathology } & \multicolumn{2}{|c|}{ Laparoscopy } \\
\hline & & Frequency & Percentage & Frequency & Percentage \\
\hline \multirow[t]{3}{*}{ Metastases $^{\ddagger}$} & Existence & 4 & 8 & 4 & 8 \\
\hline & Absence & 46 & 92 & 46 & 92 \\
\hline & Indistinguishable & 0 & 0 & 0 & 0 \\
\hline \multirow[t]{3}{*}{ Lymph node involvement II } & Existence & 25 & 50 & 36 & 72 \\
\hline & Absence & 21 & 42 & 14 & 28 \\
\hline & Indistinguishable ${ }^{\dagger}$ & 4 & 8 & 0 & 0 \\
\hline \multirow[t]{6}{*}{ Depth of Tumor Penetration ${ }^{\circ}$} & T0 & 1 & 2 & 0 & 0 \\
\hline & $\mathrm{T} 1$ & 7 & 14 & 0 & 0 \\
\hline & $\mathrm{T} 2$ & 9 & 18 & 0 & 0 \\
\hline & T3 & 22 & 44 & 4 & 8 \\
\hline & $\mathrm{T} 4$ & 7 & 14 & 5 & 10 \\
\hline & Unsuccessful & $4^{\Delta}$ & 8 & 41 & 82 \\
\hline
\end{tabular}

\footnotetext{
₹ Metastasis is the presence of visceral or intra-abdominal involvement. II Lymph node involvement mean the presence of abdominal lymph nodes involvement. † Refers to four patients in which the metastasis was confirmed in the laparoscopic stage and therefore the pathological report was not available for the surgical specimen.

${ }^{\circ}$ The amount of tumor invasion to different tissue layers of the patients, the results are as follows:

T1: tumor invasion to Lamina propria, muscularis mucosa or sub mucosa

T2: tumor invasion to muscularis propria

T3: Transmission of the tumor from subserousal connective tissue without invasion to peritoneum or adjacent structures T4: tumor invasion to adjacent structures

$\Delta$ Refers to four patients in which the metastasis was confirmed in the laparoscopic stage and therefore the pathological report was not available to determine the depth of tumor penetration.
}

years, which these findings corresponds to the previous studies that reported the $6^{\text {th }}$ or $7^{\text {th }}$ decades of life are the highest probability of the incidence of the disease [2]. In general, according to the findings, it is important to pay close attention to the upper gastrointestinal symptoms in patients referring to clinics in the $6^{\text {th }}$ or $7^{\text {th }}$ decades. Also, paying more attention to the patients` complaints, risk factors and family history of the patients in this age should be considered by all medical groups. The need for quicker referral to gastrointestinal services is required for further evaluation and, in specific cases; screening activities are required according to the existing guidelines.

In addition, the location of the tumor, as referred to in the new sources, seems to be increasing in the proximal portion of the stomach. According to available sources, about $30 \%$ of gastric tumors are located in one third of the distal, $20 \%$ of the tumors are in the middle part and about $40 \%$ are related to the proximal area of stomach. According to the statistics, $10 \%$ of the tumors involve the stomach as a whole [5]. In the present study, 21 patients (42\%) had tumors in one-third distal, 17 patients (34\%) had tumors in one-third proximal and 12 patients (24\%) had tumors in middle-third portion of the stomach. The gastric cancer staging methods are used according to the specific features and limitations of each method. In general, none of these methods seems to have the same sensitivity and specificity in the staging of all three options, T, N, M. Laparoscopy can be a good way to diagnose metastasis in upper gastrointestinal cancers before laparotomy, and it can prevent unnecessary surgeries and help in choosing the right treatment method [1]. In the present study, laparoscopy in the evaluation of lymph node involvement revealed a sensitivity of $84 \%$ and a specificity of $47.62 \%$. In valid sources, the sensitivity and specificity of laparoscopy for the detection of lymph node involvement were $54.5 \%$ and $100 \%$, respectively [16]. It is worth noting $s$ that most of the previous studies have compared the two methods of CT-Scan and 
Laparoscopy finding statistically significant differences. In this study, sensitivity, specificity, positive predictive value and negative predictive value of laparoscopy were $100 \%$ in metastasis assessment. In this study, the results of laparoscopic tumor staging were compared with the pathology report of the surgical specimen. Laparoscopy is basically unable to detect stages 1, 2 and even 3 of tumor depths (T) due to the technical limitations. Subsequently it's not worth it in this regard.

In addition, direct to the descriptive results of gastric tumor staging in the present study, reveals some remarkable points; including the fact that the highest percentages of patients are in the high risk group which indicates a delay in the diagnosis of gastric cancer or in a simpler way, late referring of patients to doctors. Also, due to the presence of visceral metastases in high risk group with $\leq \mathrm{N} 3$, performing preoperative laparoscopy seem logical in the high risk group (N+/T3-T4), especially in patients with $\leq \mathrm{N} 2-[\mathrm{N} 3 \mid$. Based on the results of this study, the use of diagnostic laparoscopy in the high risk group with $\leq \mathrm{N} 2$ in $16 \%$ of patients has changed the pathway of treatment, in $30 \%$ of $\leq \mathrm{N} 3$ cases, changed the process of the patient's treatment and prevented unnecessary laparotomy; these are of considerable benefits as of using laparoscopy.

\section{Conclusion}

Regarding the capabilities of CT scan and laparoscopy, the use of a step-by-step combination method in gastric cancer staging is a logical one. In fact, none of these methods single-handedly are unable to provide a reliable physician confidence in the tumor stage to determine the type of treatment. Hence, at least two combination methods should be used. Although applying both CT and Laparoscopic methods can be done at the same time, it has a limitation regarding economic aspect and it has problem to access to a diagnostic method. Therefore, it is recommended to perform diagnostic laparoscopy before surgery for high risk patients for metastasis $(\mathrm{N}+$ / T3-T4).

Conflict of interests: Authors state no conflict of interest.

\section{References}

1. Chen WQ, Zheng RS, Zhang SW, Zeng HM, Zou XN, He J. Analysis of cancer incidence and mortality in elderly population in China, 2013; Zhonghua Zhong Liu Za Zhi. Chin J Oncol. 2017;39(1):60-6.

2. Ghanadi K, Anbari Kh, Obeidavi Z, Beiranvand B, Almasian M, Beiki O. Risk og Gastric Cancer Associated with Family History of other Cancers of others Cancers of the Digestive System. BCCR. 2014;6(3):30-42.

3. Akbari MA, Mohamadi G. Cancers of Womens in Iran. $1^{\text {st }}$ ed. Tehran. Mohsen Publication. 2014. [In Persian].

4. American Joint Committee on Cancer (AJCC), $7^{\text {th }}$ Edition 2010. TNM staging for gastric cancer. Available from http://www. uptodate.com/2015UpTodate.

5. Karpeh MS. Staging Laparoscopy for Gastric Cancer. Operative Techniques in General Surgery. 2003;5:50-4.

6. Karimi P, Islami F, Anandasabapathy S, Freedman ND, Kamangar F. Gastric cancer: descriptive epidemiology, risk factors, screening, and prevention. Cancer Epidemiol Biomarkers Prev. 2014;23(5):700-713.

7. Yoon $\mathrm{H}$, Lee $\mathrm{DH}$. New approaches to gastric cancer staging: beyond EUS, CT and PET. WJG. 2014;20(38):13783-13790.

8. Power DG, Schattner MA, Gerdes H, Brener B, Markowitz AJ, Capanu M, et al. EUS can improve the selection for laparoscopy in patients with localized gastric cancer. J Am Surg. 2009;208:173-178.

9. Karanicolas PJ, Elkin EB, Jack LM, Atona CL, Strong VE, Brennan MF, et al. Staging Laparoscopy in the management of gastric cancer: A population-based analysis. Journal of the American College of Surgeons. 2011;213(5):644-651.

10. Burbidge S, Mahady K, Naik K. The role of CT and staging laparoscopy in the staging of gastric cancer. Clinical Radiology. 2013;68:251-255.

11. Feldman M, Friedman LS, Brandt LJ. Gastrointestinal and liver disease. Sleisenger and Fordtran,s. $9^{\text {th }}$ Edition 2010.Vol 1.900902,622 .

12. Dixon M, Cardoso R, Tinmouth J, Helyer L, Law C, Swallow $C$, et al. What studies are necessary for staging gastric adenocarcinoma? Results of an international RAND/UCLA expert panel. Gastric Cancer. 2014;17:377-382.

13. Somi MH, Golzari M, Farhang S, Naghashi S, Abdollahi L. Gastrointestinal cancer incidence in East Azerbaijan, Iran: Update on 5 Year incidence and trends. Asian Pac J Cancer Prev. 2014;15(9):3945-3949.

14. NCCN Clinical Practice Guideline in Oncology (NCCN Guidelines), Gastric Cancer. Version 3. 2015.

15. Japanese Gastric Cancer Association. Japanese Classification of Gastric Carcinoma - $2^{\text {nd }}$ English Edition. Gastric Cancer. 1998;1(1):10-24.

16. Zinner MJ, Ashley SW. Maingot's Abdominal Operations. $12^{\text {th }}$ Edition, 2013:80-82,463-467. 